\title{
ANALYTICAL TRANSIENT ANALYSIS OF LAYERED COMPOSITE MEDIUM SUBJECTED TO DYNAMIC INPLANE IMPACT LOADINGS
}

\author{
CHIEN-CHING MA and KUO-CHENG HUANG \\ Department of Mechanical Engineering, National Taiwan University, Taipei, Taiwan 10617 , \\ Republic of China \\ (Received 13 September 1994 ; in revised form 4 October 1995)
}

\begin{abstract}
In this study, the dynamic transient response of a layered composite with $n$ layers under dynamic inplane impact loading on the surface is investigated in detail. One of the objectives of this study is to develop an effective analytical method for determining transient solutions. Complete solution of the contribution for each wave is expressed in an explicit form. These solutions will provide reliable solutions that can guide the development of numerical methods. In analyzing this problem, the waves reflected and refracted by the interfaces within the composite must be taken into account, which will generate infinite waves on each composite layer and make the problem extremely complicated to analyze. The problem is solved by using the Laplace transform method and the inverse transform is evaluated by means of Cagniard's method. The transient responses of stresses and displacement in the transform domain on each layer are expressed in a closed form. The equation for each wave front propagating in the layered composite is determined in this study. For the numerical investigation of the transient response of stress, a two layered composite medium is analyzed in detail. Copyright $(1996$ Elsevier Science Ltd.
\end{abstract}

\section{INTRODUCTION}

An elastic half space is the simplest model for studying the effect of a free surface on the propagation of waves generated by the buried or surface source (detailed surveys of the literature on this subject are given by Ewing et al. (1957), Achenbach (1975), Pao and Gajewski (1977), Miklowitz (1978), and Aki and Richards (1980)). The classical analysis in this area was given by Lamb (1904) who treated four basic problems, the surface normal line and point load sources, and the buried line and point sources of dilatation. Since this early analysis of Lamb, many contributions have appeared, pertaining to what is commonly referred to as Lamb's problem. Lamb (1904) established also the method to evaluate inverse Laplace transforms. The method was later developed by Cagniard (1939) and independently by Pekeris (1940). Substantial simplification of the method was provided by de Hoop (1960). The method is usually referred to as the Cagniard-de Hoop method. The solution for a seismic pulse applied on the surface of a half space was completed by Pekeris (1955a) and that for a horizontal dynamic force by Chao (1960). Buried source problems are of considerable interest in seismology and have been studied by many investigators including Lamb (1904), Pekeris (1955b), Pekeris and Lifson (1957), Pekeris and Longman (1958), and Borejko (1987). An approach to the buried line source problem based on the method of Cagniard (1939) was applied by Garvin (1956). Recently, Tsai and Ma (1991) obtained the complete transient solutions of buried dynamic point forces and displacement jumps for an elastic half space. Ma and Huang (1996) have constructed the exact transient solutions of buried dynamic forces for elastic bimaterial problem. The solution is more complex when additional plane boundaries are introduced to form a layered half space. The problem of transient wave propagation in a multilayered solid can be studied by the method of generalized ray (see Pao and Gajewski (1977), and Borejko and Ziegler (1991)).

The applications of composite materials have grown very rapidly for the last two decades because of their high strength and light weight. The methods to find the response of composite laminates under various conditions have appeared to be of most significant concern in this field. The capability of composite materials to transmit impact loads is often the critical feature of design. For static analysis, this has already received widespread 
attention in the last two decades. However, the study of transient response has started to grow in recent years. Hence, the characterization and methodology for studying wave propagation in composite materials is of prime interest.

Wave propagation in layered media has been investigated extensively by many people because of its wide applications. A key to attacking these problems involves considering the average stress and strain response to waves whose wavelengths are long compared with the characteristic dimension of the layer. Such an approach was first proposed and developed by Sun et al. (1968) and they have named it the "effective modulus" theory. This theory is based on the assumption that the layered medum behaves, on the whole, as a homogeneous transversely isotropic continuum. Harmonic waves in composites with isotropic layers were studied by Stern et al. (1971), Hegemier and Nayfeh (1973), Hegemier and Bache (1973), Drumheller and Bedford (1973), and Nemat-Nasser and Yamada (1981). Transient plane waves propagating in a periodically layered elastic medium were examined by Ting and Mukunoki (1979), Mukunoki and Ting (1980), and Tang and Ting (1985). In studying the wave propagation in a layered medium, various approximate theories have been proposed to predict the dynamic response. Most of the approximate theories can predict satisfactorily the frequency equation due to a sinusoidal oscillation and some can also predict the late-time asymptotic solution in a semi-infinite layered medium due to a step load applied at the boundary.

Analytical investigation of the layered composite medium subjected to impact loading has been meagre because the transient analysis involves numerous parameters and is enormously complex. The stress wave solution is not only lime-dependent but it interacts with the material properties of the constituents of the composite and the various geometric parameters. Only few researches used the transient analysis to study wave propagation in a multilayered solid (see, for example, Pao and Gajewski (1977), and Borejko and Ziegler (1991)). For transient waves, one could in theory obtain the solution by superimposing harmonic waves of all frequencies and all modes. This approach, however, is not practical and a more direct approach should be employed to study the transient waves. Most of the investigations on layered media are focused on the case of periodic layering, there are only very limited studies analyzing the behavior of waves in randomly layered media. In the present paper, the transient response due to a concentrated inplane dynamic point force at the top surface of an $n$ layered composite medium is investigated. The material properties and the thickness are different in each layer. The derivation of the formal solution in the form of an infinite series is presented. The analytical solution obtained here is exact and it is expressed in a simple closed form ; each term of the solution represents a physical transient wave. For the numerical examples, a two layered composite medium is considered. The transient stresses at different locations in the two layered composite are presented.

\section{FORMULATION OF THE PROBLEM}

Consider a stress-free composite medium of $n$ layers with parallel plane boundaries, each layer differing in material properties and thickness, as shown in Fig. 1. Each layer is assumed homogeneous and isotropic and perfect bounding is considered at the interfaces. Let layer 1 denote the top layer on which the dynamic loading is applied and layer $n$ the bottom layer. A Cartesian coordinate system is oriented so that the $y$-axis is normal to the layers with the $x, z$-axis in the plane of the layers, as shown in Fig. 1. Only motion in the $x-y$ plane is to be considered. The top face of the $n$ layered medium lies in the plane $y=0$ and $-\infty<x<\infty$. At time $t=0$, a dynamic concentrated inplane force of magnitude $\sigma_{0}$ (per unit length in the $z$-direction) having step-function dependence on time acts at $x=0$, $y=0$. The boundary conditions on top and bottom layers of the problem can be written as

$$
\begin{gathered}
\sigma_{y y}^{(1)}(x, 0, t)=\sigma_{0} \delta(x) H(t) \text { for }-\infty<x<\infty, \\
\sigma_{y y}^{(n)}\left(x,-h^{(n)}, t\right)=0 \text { for } \quad-\infty<x<\infty,
\end{gathered}
$$




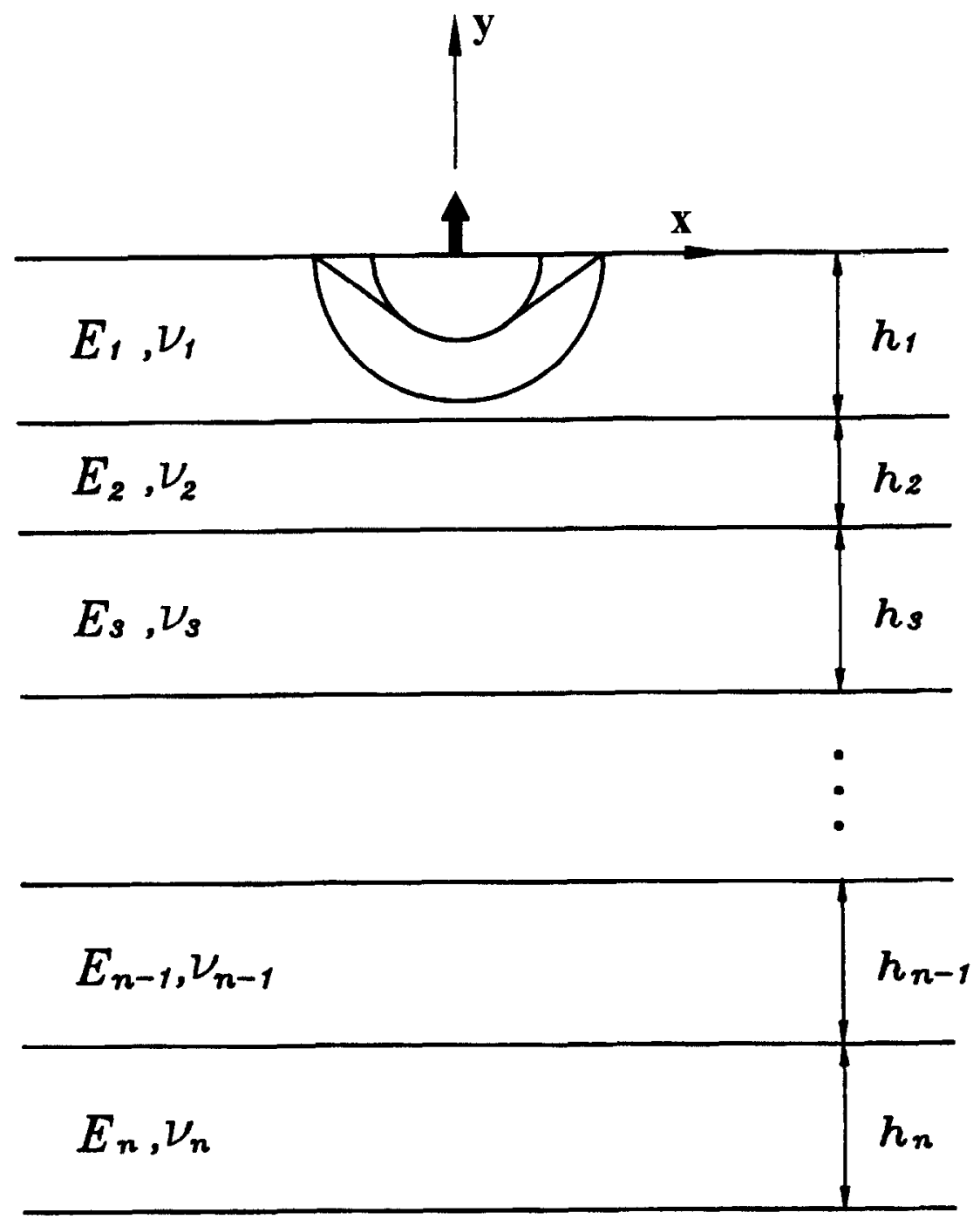

Fig. 1. Configuration and coordinate system of an $n$ layered composite medium.

$$
\begin{gathered}
\sigma_{x y}^{(1)}(x, 0, t)=0 \text { for }-\infty<x<\infty, \\
\sigma_{x y}^{(n)}\left(x,-h^{(n)}, t\right)=0 \text { for }-\infty<x<\infty,
\end{gathered}
$$

where

$$
h^{(n)}=\sum_{j=1}^{n} h_{j}
$$

in which $h_{j}$ is the thickness of the $j$ th layer, $\delta$ is the delta function and $H$ is the Heaviside step function. Perfect bonding along the interface ensures the stress and displacement continuity across each interface.

$$
\begin{array}{ll}
\sigma_{y y}^{(i)}\left(x,-h^{(i)}, t\right)=\sigma_{y y}^{(i+1)}\left(x,-h^{(i)}, t\right) & 1 \leqslant i \leqslant n-1, \\
\sigma_{x y}^{(i)}\left(x,-h^{(i)}, t\right)=\sigma_{x y}^{(i+1)}\left(x,-h^{(i)}, t\right) & 1 \leqslant i \leqslant n-1, \\
u^{(i)}\left(x,-h^{(i)}, t\right)=u^{(i+1)}\left(x,-h^{(i)}, t\right) & 1 \leqslant i \leqslant n-1, \\
v^{(i)}\left(x,-h^{(i)}, t\right)=v^{(i+1)}\left(x,-h^{(i)}, t\right) & 1 \leqslant i \leqslant n-1 .
\end{array}
$$


where the superscripts denote the $i$ th layer and $(i+1)$ th layer. In the absence of body forces, the two-dimensional equations of motion, expressed in terms of two displacement potentials $\phi$ and $\psi$, are

$$
\begin{aligned}
& \frac{\partial^{2} \phi}{\partial x^{2}}+\frac{\partial^{2} \phi}{\partial y^{2}}=a^{2} \frac{\partial^{2} \phi}{\partial t^{2}} \\
& \frac{\partial^{2} \psi}{\partial x^{2}}+\frac{\partial^{2} \psi}{\partial y^{2}}=b^{2} \frac{\partial^{2} \psi}{\partial t^{2}}
\end{aligned}
$$

where

$$
\begin{aligned}
& a=\sqrt{\frac{\rho}{\Lambda+2 \mu}}=\frac{1}{C_{L}}, \\
& b=\sqrt{\frac{\rho}{\mu}}=\frac{1}{C_{S}} .
\end{aligned}
$$

Here $\mu$ and $\rho$ are the shear modulus and the mass density of the material, respectively, $\Lambda$ is the Lamé constant, $a$ and $b$ are the slownesses of longitudinal and shear waves. Note that eqns (9) and (10) are uncoupled. The $\phi$ and $\psi$ are also referred to as the longitudinal and shear wave potentials, respectively. The displacement-potential relations are

$$
\begin{aligned}
& u=\frac{\partial \phi}{\partial x}+\frac{\partial \psi}{\partial y}, \\
& v=\frac{\partial \phi}{\partial y}-\frac{\partial \psi}{\partial x},
\end{aligned}
$$

where $u$ and $v$ are the displacements in the $x$ and $y$ directions, respectively. On invoking Hooke's law, the stresses are

$$
\begin{gathered}
\sigma_{x x}=\Lambda\left(\frac{\partial^{2} \phi}{\partial x^{2}}+\frac{\partial^{2} \phi}{\partial y^{2}}\right)+2 \mu\left(\frac{\partial^{2} \phi}{\partial x^{2}}+\frac{\partial^{2} \psi}{\partial x \partial y}\right) \\
\sigma_{v y}=\Lambda\left(\frac{\partial^{2} \phi}{\partial x^{2}}+\frac{\partial^{2} \phi}{\partial y^{2}}\right)+2 \mu\left(\frac{\partial^{2} \phi}{\partial y^{2}}-\frac{\partial^{2} \psi}{\partial x \partial y}\right), \\
\sigma_{x y}=\mu\left(2 \frac{\partial^{2} \phi}{\partial x \partial y}-\frac{\partial^{2} \psi}{\partial x^{2}}+\frac{\partial^{2} \psi}{\partial y^{2}}\right) .
\end{gathered}
$$

The one-sided Laplace transform over time and the bilateral Laplace transform on the spatial variable $x$ for a function $\phi$ are defined by

$$
\hat{\phi}(\lambda, y, p)=\int_{-\infty}^{\infty} \mathrm{e}^{-p \lambda x} \int_{0}^{\infty} \phi(x, y, t) \mathrm{e}^{-p t} \mathrm{~d} t \mathrm{~d} x,
$$

where $p$ is a positive real number, large enough to ensure convergence of the integral, and $\lambda$ is a complex variable. The solutions of eqns (9) and (10) in the Laplace transform domain are given in layer $(i)$ by 


$$
\begin{gathered}
\hat{\phi}^{(i)}=A_{i} \mathrm{e}^{p x_{j} y}+B_{i}^{-p \alpha_{i} y}, \quad i=1, \ldots, n, \\
\psi^{(i)}=C_{i} \mathrm{e}^{p \beta_{i} y}+D_{i} \mathrm{e}^{-p \beta_{i} y}, \quad i=1, \ldots, n,
\end{gathered}
$$

where

$$
\alpha_{i}=\sqrt{a_{i}^{2}-\lambda^{2}}, \quad \beta_{i}=\sqrt{b_{i}^{2}-\lambda^{2}} .
$$

The coefficients $A_{i}, B_{i}, C_{i}$, and $D_{i}$ are to be determined through the transforms of the boundary and continuity conditions. The condition $\operatorname{Re} \alpha_{i} \geqslant 0\left(\operatorname{Re} \beta_{i} \geqslant 0\right)$ is satisfied by providing branch cuts along $a_{i} \leqslant|\operatorname{Re} \lambda|<\infty\left(\mathrm{b}_{i} \leqslant|\operatorname{Re} \lambda|<\infty\right), \operatorname{Im} \lambda=0$, and choosing the branch of positive square roots.

\section{TRANSIENT SOLUTIONS IN TRANSFORM DOMAIN FOR THE $n$ LAYERED MEDIUM}

This section is concerned with the derivation of the undetermined coefficients $A_{i}, B_{i}$, $C_{i}$, and $D_{i}$ expressed in (16) and (17). The relationship of unknown constants for the same layer (i.e., (18)) and for the different layer (i.e., (19)) can be expressed as follows:

$$
\begin{gathered}
{\left[\begin{array}{l}
B_{i} \\
D_{i}
\end{array}\right]=\mathbf{T}_{i}\left[\begin{array}{l}
A_{i} \\
C_{i}
\end{array}\right],} \\
{\left[\begin{array}{c}
A_{i+1} \\
C_{i+1}
\end{array}\right]=\mathbf{G}_{i / i+1}\left[\begin{array}{l}
A_{i} \\
C_{i}
\end{array}\right],}
\end{gathered}
$$

where $\mathbf{T}_{i}$ and $\mathbf{G}_{i / i+1}$ are $2 \times 2$ matrices, determined by the boundary and continuity conditions. We also have

$$
\left[\begin{array}{c}
B_{i} \\
D_{i}
\end{array}\right]=\mathbf{H}_{i / l+1}\left[\begin{array}{c}
A_{i} \\
C_{i}
\end{array}\right]+\mathbf{F}_{i+1 / i}\left[\begin{array}{c}
B_{i+1} \\
D_{i+1}
\end{array}\right],
$$

where $\mathbf{H}_{i / i+1}$ and $\mathbf{F}_{i+1 / i}$ are $2 \times 2$ matrices, called the reflected potential matrix and the refracted potential matrix, respectively. From (18), (19), and (20), $\mathbf{T}_{i}$ can be written as follows

$$
\mathbf{T}_{i}=\mathbf{H}_{i / i+1}+\mathbf{F}_{i+1 / i} \mathbf{T}_{i+1} \mathbf{G}_{i / i+1}
$$

The expression

$$
\left[\begin{array}{c}
A_{i+1} \\
C_{i+1}
\end{array}\right]=\mathbf{H}_{i+1 / i}\left[\begin{array}{c}
B_{i+1} \\
D_{i+1}
\end{array}\right]+\mathbf{F}_{i / i+1}\left[\begin{array}{c}
A_{i} \\
C_{i}
\end{array}\right]
$$

can be written as

$$
\left[\begin{array}{c}
A_{i+1} \\
C_{i+1}
\end{array}\right]=\sum_{i=0}^{\infty}\left(\mathbf{H}_{i+1 / i} \mathbf{T}_{i+1}\right)^{l} \mathbf{F}_{i / i+1}\left[\begin{array}{c}
A_{i} \\
C_{i}
\end{array}\right]
$$

Hence, we get 


$$
\begin{gathered}
\mathbf{G}_{i / i+1}=\sum_{i=\mathbf{0}}^{\infty}\left(\mathbf{H}_{i+1 / i} \mathbf{T}_{i+1}\right)^{l} \mathbf{F}_{i / i+1}, \\
\mathbf{T}_{i}=\mathbf{H}_{i / i+1}+\mathbf{F}_{i+1 / i} \mathbf{T}_{i+1} \sum_{i=0}^{\infty}\left(\mathbf{H}_{i+1 / i} \mathbf{T}_{i+1}\right)^{l} \mathbf{F}_{i / i+1} .
\end{gathered}
$$

The reflected potential matrix $\mathbf{H}_{i j}$ can be expressed as

$$
\mathbf{H}_{i / j}=\frac{1}{R_{i j j}}\left[\begin{array}{cc}
\Theta_{1} \Phi_{1} & \Theta_{2} \Phi_{2} \\
\Theta_{3} \Phi_{3} & \Theta_{4} \Phi_{4}
\end{array}\right],
$$

where

$$
\begin{aligned}
R_{i / j}=4 \lambda \alpha_{i} \alpha_{j} \beta_{i} \beta_{j}(1-\gamma)^{2}+\lambda^{2}\left[\beta_{i}^{2}-(1-\gamma) \lambda^{2}-\gamma \beta_{j}^{2}\right]^{2}+\gamma\left(\alpha_{i} \beta_{j}+\alpha_{j} \beta_{i}\right) b_{i}^{2} b_{j}^{2} \\
+\alpha_{i} \beta_{i}\left[\gamma \beta_{j}^{2}+\lambda^{2}(2-\gamma)\right]^{2}+\alpha_{j} \beta_{j}\left[\beta_{i}^{2}+(2 \gamma-1) \lambda^{2}\right]^{2}, \quad(i, j \text { no summation })
\end{aligned}
$$

in which

$$
\gamma=\mu_{j} / \mu_{i},
$$

and

$$
\begin{aligned}
& \Theta_{1}(\lambda)=\left\{4 \lambda^{2} \alpha_{i} \alpha_{j} \beta_{i} \beta_{j}(1-\gamma)^{2}-\lambda^{2}\left(\beta_{i}^{2}-\lambda^{2}-\gamma \beta_{j}^{2}+\gamma \hat{\lambda}^{2}\right)^{2}\right. \\
& \left.+\gamma\left(\alpha_{i} \beta_{j} \quad \alpha_{j} \beta_{i}\right) b_{i}^{2} b_{j}^{2}+\alpha_{i} \beta_{i}\left(\gamma \beta_{j}^{2}-\gamma \lambda^{2}+2 \lambda^{2}\right)^{2}-\alpha_{j} \beta_{j}\left(\beta_{i}^{2}-\lambda^{2}+2 \gamma \lambda^{2}\right)^{2}\right\}, \\
& \Theta_{2}(\lambda)=\left\{4 \lambda \alpha_{j} \beta_{i} \beta_{j}(1-\gamma)\left(\beta_{i}^{2}-\lambda^{2}+2 \gamma \lambda^{2}\right)-2 \beta_{i} \lambda\left(\gamma \beta_{j}^{2}-\gamma \lambda^{2}-\beta_{i}^{2}+\lambda^{2}\right)\left(\gamma \beta_{j}^{2}-\gamma \lambda^{2}+2 \lambda^{2}\right)\right\}, \\
& \Theta_{3}(\lambda)=\left\{4 \lambda \alpha_{i} \alpha_{j} \beta_{j}(1-\gamma)\left(\beta_{i}^{2}-\lambda^{2}+2 \gamma \lambda^{2}\right)-2 \alpha_{i} \lambda\left(\gamma \beta_{j}^{2}-\gamma \lambda^{2}+2 \lambda^{2}\right)\left(\gamma \beta_{j}^{2}-\beta_{i}^{2}+\lambda^{2}-\gamma \lambda^{2}\right)\right\}, \\
& \Theta_{4}(\lambda)=\left\{4 \lambda^{2} \alpha_{i} \alpha_{j} \beta_{i} \beta_{j}(1-\gamma)^{2}-\lambda^{2}\left(\beta_{i}^{2}-\lambda^{2}-\gamma \beta_{j}^{2}+\gamma \lambda^{2}\right)^{2}\right. \\
& \left.+\gamma\left(\alpha_{j} \beta_{i}-\alpha_{i} \beta_{j}\right) b_{j}^{2} b_{i}^{2}+\alpha_{i} \beta_{i}\left(\gamma \beta_{j}^{2}-\gamma \lambda^{2}+2 \lambda^{2}\right)^{2}-\alpha_{j} \beta_{j}\left(\beta_{i}^{2}-\lambda^{2}+2 \gamma \lambda^{2}\right)^{2}\right\}, \\
& \Phi_{1}= \begin{cases}\mathrm{e}^{-2 p \alpha_{i} h^{(i)}} & \text { if } \quad i<j \\
\mathrm{e}^{2 p x_{i} h^{(j)}} & \text { if } j<i,\end{cases} \\
& \Phi_{2}=\left\{\begin{array}{lll}
\mathrm{e}^{-p\left(\alpha_{i}+\beta_{i}\right) h^{(i)}} & \text { if } \quad i<j \\
-\mathrm{e}^{p\left(a_{i}+\beta_{i}\right) h^{(i)}} & \text { if } \quad j<i,
\end{array}\right. \\
& \Phi_{3}=\left\{\begin{array}{lll}
\mathrm{e}^{-p\left(\alpha_{i}+\beta_{j}\right) h^{(i)}} & \text { if } \quad i<j \\
\mathrm{e}^{p\left(\alpha_{i}+\beta_{i}\right) h^{(i)}} & \text { if } \quad i>j,
\end{array}\right. \\
& \Phi_{4}= \begin{cases}\mathrm{e}^{2 p \beta h^{(i)}} & \text { if } i<j \\
\mathrm{e}^{2 p \beta_{i} h^{(j)}} & \text { if } j<i .\end{cases}
\end{aligned}
$$

The function $R_{i / j}$ in (26) is the characteristic function for Stoneley waves at the interface of two dissimilar solids. Note that there are combinations of adjacent materials that admit the Stoneley wave and others that do not (see Chadwick and Borejko (1994)). When the solid $j$ is absent, the function $R_{i / j}$ will reduce to the equation of Rayleigh surface wave. The refracted wave potential $\mathbf{F}_{i, j}$ can be expressed as

$$
\mathbf{F}_{i j j}=\frac{1}{R_{i j j}}\left[\begin{array}{cc}
\Theta_{5} \Phi_{5} & \Theta_{6} \Phi_{6} \\
\Theta_{7} \Phi_{7} & \Theta_{8} \Phi_{8}
\end{array}\right]
$$

where 


$$
\begin{aligned}
& \Theta_{5}(\lambda)=2 \alpha_{i} b_{i}^{2}\left\{\beta_{j}\left(\beta_{i}^{2}-\lambda^{2}+2 \gamma \lambda^{2}\right)+\beta_{i}\left(\gamma \beta_{j}^{2}-\gamma \lambda^{2}+2 \lambda^{2}\right)\right\}, \\
& \Theta_{6}(\lambda)=2 \beta_{i} \lambda b_{i}^{2}\left\{2 \alpha_{i} \beta_{j}(1-\gamma)+\gamma \beta_{j}^{2}-\gamma \lambda^{2}-\beta_{i}^{2}+\lambda^{2}\right\}, \\
& \Theta_{7}(\lambda)=2 \alpha_{i} \lambda b_{i}^{2}\left\{2 \alpha_{j} \beta_{i}(1-\gamma)+\gamma \beta_{j}^{2}-\gamma \lambda^{2}-\beta_{i}^{2}+\lambda^{2}\right\}, \\
& \Theta_{8}(\lambda)=2 \beta_{i} b_{i}^{2}\left\{\alpha_{j}\left(\beta_{i}^{2}-\lambda^{2}+2 \gamma \lambda^{2}\right)+\alpha_{i}\left(\gamma \beta_{j}^{2}-\gamma \lambda^{2}+2 \lambda^{2}\right)\right\},
\end{aligned}
$$

in which

$$
\begin{aligned}
& \Phi_{5}=\left\{\begin{array}{lll}
\mathrm{e}^{p\left(\alpha_{j}-\alpha_{i}\right) h^{(i)}} & \text { if } & i<j \\
\mathrm{e}^{p\left(x_{i}-\alpha_{j}\right) h^{(j)}} & \text { if } & j<i,
\end{array}\right. \\
& \Phi_{6}=\left\{\begin{array}{lll}
-\mathrm{e}^{p\left(x_{j}-\beta_{i}\right) h^{(i)}} & \text { if } & i<j \\
\mathrm{e}^{p\left(\beta_{i}-\alpha_{j}\right) h^{(j)}} & \text { if } j<i,
\end{array}\right. \\
& \Phi_{7}=\left\{\begin{array}{lll}
\mathrm{e}^{p\left(x_{j}-\beta_{j}\right) h^{(i)}} & \text { if } \quad i>j \\
-\mathrm{e}^{p\left(\beta_{i}-x_{j}\right) h^{(j)}} & \text { if } j<i,
\end{array}\right. \\
& \Phi_{8}=\left\{\begin{array}{lll}
\mathrm{e}^{p\left(\beta_{j}-\beta_{i}\right) h^{(i)}} & \text { if } \quad i<j \\
\mathrm{e}^{p\left(\beta_{i}-\beta_{j}\right) h^{(j)}} & \text { if } \quad j<i .
\end{array}\right.
\end{aligned}
$$

From the boundary condition of the $n$th layer, we have

$$
\mathbf{T}_{n}=\mathbf{H}_{n, n+1},
$$

where

$$
\mathbf{H}_{n / n+1}=\frac{1}{R_{n}}\left[\begin{array}{cc}
{\left[4 \lambda^{2} \alpha_{n} \beta_{n}-\left(\beta_{n}^{2}-\lambda^{2}\right)^{2}\right] \mathrm{e}^{-2 p \alpha_{n} h^{(n)}}} & -4 \lambda \beta_{n}\left(\beta_{n}^{2}-\lambda^{2}\right) \mathrm{e}^{-p\left(\alpha_{n}+\beta_{n}\right) h^{(n)}} \\
4 \lambda \alpha_{n}\left(\beta_{n}^{2}-\lambda^{2}\right) \mathrm{e}^{-p\left(\alpha_{n}+\beta_{n}\right) h^{(n)}} & {\left[4 \lambda^{2} \alpha_{n} \beta_{n}-\left(\beta_{n}^{2}-\lambda^{2}\right)^{2}\right] \mathrm{e}^{-2 p \beta_{n} h^{\left(n^{n}\right)}}}
\end{array}\right],
$$

in which

$$
R_{n}=4 \lambda^{2} \alpha_{n} \beta_{n}+\left(\beta_{n}^{2}-\lambda^{2}\right)^{2}
$$

From the boundary condition of the first layer, we can obtain

$$
\left[\begin{array}{l}
A_{1} \\
C_{1}
\end{array}\right]=\sum_{j=0}^{\infty}\left(\mathbf{H}_{1 / 0} \mathbf{T}_{1}\right)^{j}\left[\begin{array}{c}
\left(\beta_{1}^{2}-\lambda^{2}\right) / p^{3} \mu_{1} R_{1} \\
-2 \lambda \alpha_{1} / p^{3} \mu_{1} R_{1}
\end{array}\right]
$$

where

$$
\mathbf{H}_{1 / 0}=\frac{1}{R_{1}}\left[\begin{array}{cc}
4 \lambda^{2} \alpha_{1} \beta_{1}-\left(\beta_{1}^{2}-\lambda^{2}\right)^{2} & -4 \lambda \beta_{1}\left(\beta_{1}^{2}-\lambda^{2}\right) \\
4 \lambda \alpha_{1}\left(\beta_{1}^{2}-\lambda^{2}\right) & 4 \lambda^{2} \alpha_{1} \beta_{1}-\left(\beta_{1}^{2}-\lambda^{2}\right)^{2}
\end{array}\right],
$$

in which

$$
R_{1}=4 \lambda^{2} \alpha_{1} \beta_{1}+\left(\beta_{1}^{2}-\lambda^{2}\right)^{2}
$$

The correspondent unknowns $A_{i}, B_{i}, C_{i}$, and $D_{i}$ for each layer can then be obtained from (18) and (22). The solutions for displacements and stresses in the Laplace transform domain in layer $(i)$ are given by 


$$
\begin{gathered}
\hat{u}^{(i)}(\lambda, y, p)=p \lambda\left(A_{i} \mathrm{e}^{p \alpha_{i} y}+B_{i} \mathrm{e}^{p \alpha_{i} y}\right)+p \beta_{i}\left(C_{i} \mathrm{e}^{p \beta_{i} y}-D_{i} \mathrm{e}^{p \beta_{j} y}\right), \\
\hat{v}^{(i)}(\lambda, y, p)=p \alpha_{i}\left(A_{i} \mathrm{e}^{p \alpha_{i} y}-B_{i} \mathrm{e}^{-p \alpha_{i} y}\right)-p \lambda\left(C_{i} \mathrm{e}^{p \beta_{i} y}+D_{i} \mathrm{e}^{-p \beta_{i} y}\right), \\
\hat{\sigma}_{\lambda x}^{(i)}(\lambda, y, p)=\Lambda p^{2} a_{i}^{2}\left(A_{i} \mathrm{e}^{p \alpha_{i} y}+B_{i} \mathrm{e}^{-p \alpha_{i} y}\right)+2 \mu p^{2} \lambda^{2}\left(A_{i} \mathrm{e}^{p \alpha_{i} y}+B_{i} \mathrm{e}^{-p x_{i} y}\right) \\
+2 \mu p^{2} \lambda \beta_{i}\left(C_{i} \mathrm{e}^{p \beta_{i} y}-D_{i} \mathrm{e}^{-p \beta_{i} y}\right), \\
\hat{\sigma}_{y y}^{(i)}(\lambda, y, p)=\Lambda p^{2} a_{i}^{2}\left(A_{i} \mathrm{e}^{p \alpha_{i} y}+B_{i} \mathrm{e}^{-p \alpha_{i} y}\right)+2 \mu p^{2} \alpha_{i}^{2}\left(A_{i} \mathrm{e}^{p \alpha_{i} y}+B_{i} \mathrm{e}^{-p x_{i} y}\right) \\
-2 \mu p^{2} \lambda \beta_{i}\left(C_{i} \mathrm{e}^{p \beta_{i} y}-D_{i} \mathrm{e}^{-p \beta_{i} y}\right), \\
\hat{\sigma}_{x y}^{(i)}(\lambda, y, p)=2 \mu p^{2} \lambda \alpha_{i}\left(A_{i} \mathrm{e}^{p \alpha_{i} y}+B_{i} \mathrm{e}^{-p \alpha_{i} y}\right)+\mu p^{2}\left(\beta_{i}^{2}-\lambda^{2}\right)\left(C_{i} \mathrm{e}^{p \beta_{i} y}+D_{i} \mathrm{e}^{-p \beta_{i} y}\right) .
\end{gathered}
$$

\section{TRANSIENT SOLUTIONS FOR THE TWO LAYERED MEDIUM}

In order to explain the methodology of constructing the infinite number of reflected and refracted waves generated in each layer, a two layered composite medium is selected. Hence, $n=2$ and the respective coefficients are

$$
\begin{gathered}
{\left[\begin{array}{l}
A_{1} \\
C_{1}
\end{array}\right]=\sum_{l=0}^{\infty}\left(\mathbf{H}_{1 / 0} \mathbf{T}_{1}\right)^{l}\left[\begin{array}{c}
\left(\beta_{1}^{2}-\lambda^{2}\right) / p^{3} \mu_{1} R_{1} \\
-2 \lambda \alpha_{1} / p^{3} \mu_{1} R_{1}
\end{array}\right],} \\
{\left[\begin{array}{l}
B_{1} \\
D_{1}
\end{array}\right]=\mathbf{I}_{1} \sum_{l=0}^{\infty}\left(\mathbf{H}_{1 / 0} \mathbf{T}_{1}\right)^{l}\left[\begin{array}{c}
\left(\beta_{1}^{2}-\lambda^{2}\right) / p^{3} \mu_{1} R_{1} \\
-2 \lambda \alpha_{1} / p^{3} \mu_{1} R_{1}
\end{array}\right],} \\
{\left[\begin{array}{l}
A_{2} \\
C_{2}
\end{array}\right]=\sum_{l=0}^{\infty}\left(\mathbf{H}_{2 / 1} \mathbf{T}_{2}\right)^{l} \mathbf{F}_{1 / 2} \sum_{j=0}^{\infty}\left(\mathbf{H}_{1 / 0} \mathbf{T}_{1}\right)^{j}\left[\begin{array}{c}
\left(\beta_{1}^{2}-\lambda^{2}\right) / p^{3} \mu_{1} R_{1} \\
-2 \lambda \alpha_{1} / p^{3} \mu_{1} R_{1}
\end{array}\right],} \\
{\left[\begin{array}{c}
B_{2} \\
D_{2}
\end{array}\right]=\mathbf{T}_{2} \sum_{l=0}^{\infty}\left(\mathbf{H}_{2 / 1} \mathbf{T}_{2}\right)^{l} \mathbf{F}_{1 / 2} \sum_{j=0}^{\infty}\left(\mathbf{H}_{1 / 0} \mathbf{T}_{1}\right)^{j}\left[\begin{array}{c}
\left(\beta_{1}^{2}-\lambda^{2}\right) / p^{3} \mu_{1} R_{1} \\
-2 \lambda \alpha_{1} / p^{3} \mu_{1} R_{1}
\end{array}\right],}
\end{gathered}
$$

where

$$
\begin{aligned}
\mathbf{T}_{1} & =\mathbf{H}_{1 / 2}+\mathbf{F}_{2 / 1} \mathbf{T}_{2} \sum_{m=0}^{\infty}\left(\mathbf{H}_{2 / 1} \mathbf{T}_{2}\right)^{m} \mathbf{F}_{1 / 2} \\
\mathbf{T}_{2} & =\mathbf{H}_{2 / 3} \\
\mathbf{G}_{1 / 2} & =\sum_{l=0}^{\infty}\left(\mathbf{H}_{2 / 1} \mathbf{T}_{2}\right)^{\prime} \mathbf{F}_{1 / 2}
\end{aligned}
$$

The $\mathbf{H}_{1 / 0}$ and $\mathbf{H}_{2 / 3}$ represent the waves reflected from the upper and lower free surface, respectively. Similarly, $\mathbf{H}_{1 / 2}$ and $\mathbf{H}_{2 / 1}$ represent the waves reflected from the interface by the waves propagating in solid 1 and solid 2 , respectively. $\mathbf{F}_{1 / 2}$ represents the refracted waves transmitted through the interface from solid 1 into solid $2, \mathbf{F}_{2 / 1}$ represents the refracted waves transmitted through the interface from layer 2 into layer 1 . Hence, using the matrix formulation given by (33)-(36), all waves propagating in the two layered composite can be identified.

Each term in (33) represents propagating waves in the first layer and with the propagation direction along the negative $y$ axis. For $l=0$ in (33), it represents the incident waves (i.e., $P$ and $S$ waves). For $l=1$, the first term (i.e., $\left.\mathbf{H}_{1 / 0} \mathbf{H}_{1 / 2}\right)$ represents the waves $(P P P$, $P P S, P S P, P S S, S P P, S P S, S S P$, and $S S S$ waves) which are reflected (i.e., $\mathbf{H}_{1 / 2}$ ) at the 
interface and then reflected (i.c., $\mathbf{H}_{1 / 0}$ ) again at the upper free surface. The second term (i.e., $\mathbf{H}_{1 / 0} \mathbf{F}_{2 / 1} \mathbf{H}_{2 / 3} \mathbf{F}_{1 / 2}$ ) represents the waves which are transmitted (i.e., $\mathbf{F}_{1 / 2}$ ) across the interface, reflected (i.e., $\mathbf{H}_{2 / 3}$ ) at the lower free surface, transmitted (i.e., $\mathbf{F}_{2 / 1}$ ) across the interface and finally reflected (i.e., $\mathbf{H}_{1 / 0}$ ) at the upper free surface. Each term in (34) represents waves propagating in the direction along the positive $y$ axis for the first layer. For $l=0$ in (34), the first term (i.e., $\mathbf{H}_{1 / 2}$ ) represents the waves ( $P P, P S, S P$, and $S S$ waves) reflected at the interface.

The similar procedure is also applied to the unknown cocfficients $A_{2}, B_{2}, C_{2}$, and $D_{2}$ in the second layer. For $l=0$ and $j=0$ in (35), the first term (i.e., $\mathbf{F}_{1 / 2}$ ) represents the refracted waves $(P / P, P / S, S / P$, and $S / S$ waves) transmitted across the interface. For $l=0$ and $j=0$ in (36), the first term (i.e., $\left.\mathbf{H}_{2 / 3} \mathbf{F}_{1 / 2}\right)$ represents the waves $(P / P P, P / P S, P / S P, P / S S, S / P P$, $S / P S, S / S P$, and $S / S S$ waves) which are transmitted (i.e., $\mathbf{F}_{1 / 2}$ ) across the interface and then reflected (i.e., $\mathbf{H}_{2 / 3}$ ) at the lower free surface in layer 2.

When we combine (33)-(36) and (31), the solution for normal stress $\sigma_{y y}$ for the first few terms (i.e., the $P, S, P P, P S, S P$, and $S S$ waves in layer 1 ; the $P / P, P / S, S / P$, and $S / S$ waves in layer 2) in the Laplace transform domain can be expressed by

$$
\begin{aligned}
& \tilde{\sigma}_{y, y}^{(1)}(x, y, p)=\frac{\sigma_{0}}{2 \pi i} \int_{\Gamma}\left\{F_{1}(\lambda) \mathrm{e}^{p \alpha_{1} y+p \lambda x}+F_{2}(\lambda) \mathrm{e}^{-p x_{1}(y+2 h)+p i x}\right. \\
& +F_{3}(\lambda) \mathrm{e}^{-p\left(x_{1} y+2 \beta_{1} h\right)+p \lambda x}+F_{4}(\lambda) \mathrm{e}^{p \beta_{1} y+p i x} \\
& \left.+F_{5}(\lambda) \mathrm{e}^{-p\left(\beta_{1} y+2 x_{1} h\right)+p \lambda x}+F_{6}(\lambda) \mathrm{e}^{-p \beta_{1}(y+2 h)+p \lambda x}+\cdots\right\} \mathrm{d} \lambda,
\end{aligned}
$$

$$
\begin{aligned}
& \tilde{\sigma}_{y y}^{(2)}(x, y, p)=\frac{\sigma_{0}}{2 \pi i} \int_{\Gamma}\left\{F_{1}^{*}(\lambda) \mathrm{e}^{p\left(x_{2} y-2 \alpha_{1} h\right)+p \lambda x}+F_{2}^{*}(\lambda) \mathrm{e}^{p\left(\alpha_{2} y-2 \beta_{1} h\right)+p \lambda x}\right. \\
& \left.+F_{3}^{*}(\lambda) \mathrm{e}^{p\left(\beta_{2} y-2 \alpha_{1} h\right)+p \lambda x}+F_{4}^{*}(\lambda) \mathrm{e}^{p\left(\beta_{2} y-2 \beta_{1} h\right)+p i x}+\cdots\right\} \mathrm{d} \lambda,
\end{aligned}
$$

where

$$
\begin{gathered}
F_{1}(\lambda)=\frac{\left(b_{1}^{2}-2 \lambda^{2}\right)^{2}}{R_{1}(\lambda)}, \quad F_{2}(\lambda)=\frac{\Theta_{1}(\lambda)\left(b_{1}^{2}-2 \lambda^{2}\right)^{2}}{R_{1}(\lambda) R_{1 / 2}(\lambda)}, \\
F_{3}=-\frac{2 \Theta_{2}(\lambda)\left(b_{1}^{2}-2 \lambda^{2}\right) \lambda \alpha_{1}}{R_{1}(\lambda) R_{1 / 2}(\lambda)}, \quad F_{4}=\frac{4 \lambda^{2} \alpha_{1} \beta_{1}}{R_{1}(\lambda)}, \\
F_{5}=\frac{2 \Theta_{3}(\lambda)\left(b_{1}^{2}-2 \lambda^{2}\right) \lambda \alpha_{1}}{R_{1}(\lambda) R_{1 / 2}(\lambda)}, \quad F_{6}=-\frac{4 \Theta_{4}(\lambda) \alpha_{1} \beta_{2} \lambda^{2}}{R_{1}(\lambda) R_{1 / 2}(\lambda)}, \\
F_{1}^{*}(\lambda)=\gamma \frac{\Theta_{5}(\lambda)\left(b_{1}^{2}-2 \lambda^{2}\right)\left(b_{2}^{2}-2 \lambda^{2}\right)}{R_{1}(\lambda) R_{1 / 2}(\lambda)}, \quad F_{2}^{*}(\lambda)=-\gamma \frac{2 \Theta_{6}(\lambda)\left(b_{1}^{2}-2 \lambda^{2}\right) \lambda \alpha_{1}}{R_{1}(\lambda) R_{1 / 2}(\lambda)}, \\
F_{3}^{*}(\lambda)=-\gamma \frac{2 \Theta_{7}(\lambda)\left(b_{1}^{2} 2 \lambda^{2}\right) \lambda \beta_{2}}{R_{1}(\lambda) R_{1 / 2}(\lambda)}, \quad F_{4}^{*}(\lambda)=\gamma \frac{4 \Theta_{8}(\lambda) \alpha_{1} \beta_{2} \lambda^{2}}{R_{1}(\lambda) R_{1 / 2}(\lambda)},
\end{gathered}
$$

in which

$$
\begin{gathered}
R_{1}(\lambda)=4 \lambda^{2} \alpha_{1} \beta_{1}+\left(\beta_{1}^{2}-\lambda^{2}\right)^{2}, \\
R_{1 / 2}(\lambda)=4 \lambda \alpha_{1} \alpha_{2} \beta_{1} \beta_{2}(1-\gamma)^{2}+\lambda^{2}\left[\beta_{1}^{2}-(1-\gamma) \lambda^{2}-\gamma \beta_{2}^{2}\right]^{2}+\gamma\left(\alpha_{1} \beta_{2}+\alpha_{2} \beta_{1}\right) b_{1}^{2} b_{2}^{2} \\
+\alpha_{1} \beta_{1}\left[\gamma \beta_{2}^{2}+\lambda^{2}(2-\lambda)\right]^{2}+\alpha_{2} \beta_{2}\left[\beta_{1}^{2}+(2 \gamma-1) \lambda^{2}\right]^{2} .
\end{gathered}
$$

Next we evaluate the inverse transforms of stress $\tilde{\sigma}_{y y}$ expressed in (37) and (38). The exact transient solution in time domain can then be found by inverting the Laplace transform by Cagniard-de Hoop method. The idea of the Cagniard-de Hoop method is to deform the path of integration in the complex $\lambda$-plane in such a manner that the inverse 
Laplace transform of the integral along the new path of integration can be obtained by inspection. From the following elementary property of the one-sided Laplace transform

$$
\mathscr{L}^{-1}\left\{\int_{t_{1}}^{\infty} \mathrm{e}^{-p t} g(t) \mathrm{d} t\right\}=g(t) H\left(t-t_{1}\right),
$$

the inversion of the two transforms (i.e., the transform in time $t$ and in the spatial variable $x$ ) can be operated at one time. The transforms are inverted by means of the modified Cagniard method due to de Hoop (1960). The inversion of the transforms depends on the relative magnitudes of the slownesses and the differences for the various cases are slight. The six cases for different slowness combinations are case 1: $a_{1}<b_{1}<a_{2}<b_{2}$, case 2: $a_{1}<a_{2}<b_{1}<b_{2}$, case 3: $a_{1}<a_{2}<b_{2}<b_{1}$, case 4: $a_{2}<a_{1}<b_{2}<b_{1}$, case 5: $a_{2}<a_{1}<b_{1}<b_{2}$, case $6: a_{2}<b_{2}<a_{1}<b_{1}$. We will discuss the Laplace inversion of stress $\sigma_{y y}$ in more detail for case $2, a_{1}<a_{2}<b_{1}<b_{2}$. The first step in the procedure is to transform the path of integration $\Gamma$ into another path such that $\tilde{\sigma}_{y y}$ may be recognized as the Laplace transform of a certain function. The first and fourth terms in (37) are the incident longitudinal and shear wave, respectively, the inversion of the two terms can be found in many text books and we will not repeat it here. For the second term in (37), we consider a path of integration in the $\lambda$-plane defined by the equation

$$
\alpha_{1}(y+2 h)-\lambda x=t
$$

where $t$ is real and positive. Equation (40) can be solved for $\lambda$ to yield

$$
\lambda_{\Gamma_{+}}\left(r_{2}, \theta_{2}, t\right)=-\frac{t}{r_{2}} \cos \theta_{2} \pm i \sqrt{\frac{t^{2}}{r_{2}^{2}}-a_{1}^{2}} \sin \theta_{2}
$$

where

$$
r_{2}^{2}=(y+2 h)^{2}+x^{2}, \quad \cos \theta_{2}=\frac{x}{r_{2}}, \quad \sin \theta_{2}=\frac{y+2 h}{r_{2}} .
$$

This represents essentially a transformation of the $t$-plane to the $\lambda$-plane which changes the path of integration $\Gamma$ to $\Gamma_{ \pm}$. In the $\lambda$-plane, (41) describes a hyperbola as shown in Fig. 2a. When $t-a_{1} r_{2}=T_{P P}$, the imaginary part of $\lambda$ vanishes, and the vertex of the hyperbola is defined by $\lambda=-a_{1} \cos \theta_{2}$. The parameter $t$ is real and varies from $a_{1} r_{2}$ to $\infty$ as $\lambda$ moves away from the real axis in either the upper or lower half-plane. Since we are considering the case $a_{1}<a_{2}$, the hyperbola does not intersect any branch cut of the integrand. The paths $\Gamma_{+}$and $\Gamma_{-}$are shown in Fig. 2a. These paths will be a suitable deformed integral contour to replace the original integration path $\Gamma$. The second term of (37) becomes

$$
\tilde{\sigma}_{y y}^{(1)}(x, y, p)=\frac{\sigma_{0}}{\pi} \int_{T_{P P}}^{\infty} \operatorname{Im}\left[F_{2}\left(\lambda_{2}\right) \frac{\partial \lambda_{2}}{\partial t}\right] \mathrm{e}^{-p t} \mathrm{~d} t,
$$

where $\lambda_{2}=\lambda_{\Gamma_{+}}\left(r_{2}, \theta_{2}, t\right)$ in (41). From (39), the inversion of the Laplace transform in the time domain is

$$
\sigma_{y y}^{(1)}(x, y, t)=\frac{\sigma_{0}}{\pi} \operatorname{Im}\left[F_{2}\left(\lambda_{2}\right) \frac{\partial \lambda_{2}}{\partial t}\right] H\left(t-T_{P P}\right) .
$$

The wave front arrives at the time $t=T_{P P}$, with the subscript $P P$ denoting the reflected longitudinal wave due to the incident longitudinal $P$ wave. The stress $\sigma_{y y}^{(1)}$ expressed in (43) takes an infinite value at the wave front which is due to the representation of the impulsive force at the surface by the idealized Heaviside function $H(t)$. If the concentrated load varies 
(a)

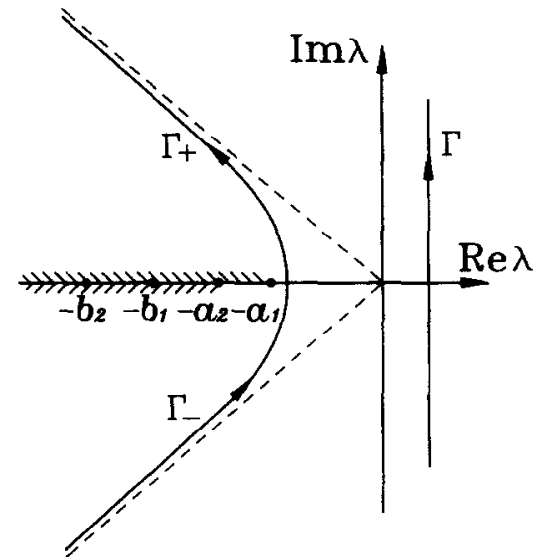

(b)

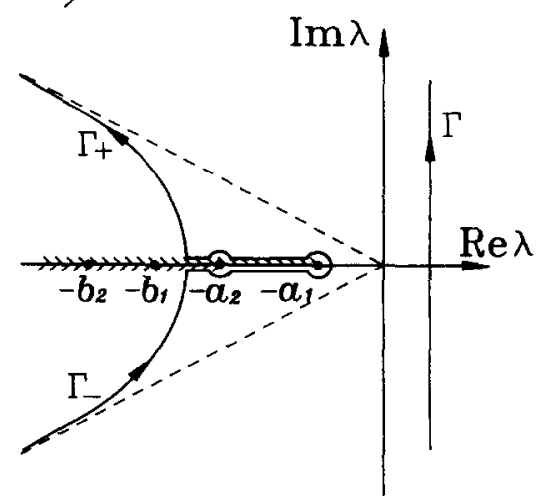

Fig. 2. (a) Integration contour as presented in (40), (44) and (48). (b) Integration contour as presented in (51).

with time as a function $f(t)$ such that $f(t=0)=0$ and $\mathrm{d} f / \mathrm{d} t(t=0)$ is continuous, then the stress will be continuous and no singularity will exist at the wave front.

As the Cagniard-de Hoop method is employed on the third term of (37), let

$$
\alpha y+2 \beta_{1} h-\lambda x=t .
$$

When the imaginary part of the root $\lambda$ in (44) vanishes, the corresponding time $t$ is denoted by $T_{S P}$. As $t$ tends from $T_{S P}$ to $\infty$, the integral path approaches the asymptote as shown in Fig. 2a. The intersection with the real axis is always located between the branch points $-a_{1}$ and $a_{1}$. The third term of (37) then becomes

$$
\tilde{\sigma}_{y y}^{(1)}(x, y, p)=\frac{\sigma_{0}}{\pi} \int_{T_{s p}}^{\infty} \operatorname{Im}\left[F_{3}\left(\lambda_{3}\right) \frac{\partial \lambda_{3}}{\partial t}\right] \mathrm{e}^{-p t} \mathrm{~d} t .
$$

The inversion of the Laplace transform is

$$
\sigma_{y y}^{(1)}(x, y, t)=\frac{\sigma_{0}}{\pi} \operatorname{Im}\left[F_{3}\left(\lambda_{3}\right) \frac{\partial \lambda_{3}}{\partial t}\right] H\left(t-T_{S P}\right),
$$

where $\lambda_{3}$ is the root of (44) and $T_{S P}$ denotes the time at which the reflected longitudinal wave front generated by the incident shear wave arrives. The $\partial \lambda_{3} / \partial t$ follows from (44) 


$$
\frac{\partial \lambda_{3}}{\partial t}=\frac{1}{-\frac{\lambda_{3} y}{\alpha_{1}}-\frac{2 \lambda_{3} h}{\beta_{1}}-x}
$$

A similar procedure is applied to the fifth term of (37), let

$$
\beta_{1} y+2 \alpha_{1} h-\lambda x=t
$$

Following the same argument as described from (44) to (47),

$$
\sigma_{y y}^{(1)}(x, y, t)=\frac{\sigma_{0}}{\pi} \operatorname{Im}\left[F_{5}\left(\lambda_{5}\right) \frac{\partial \lambda_{5}}{\partial t}\right] H\left(t-T_{P S}\right)
$$

where $\lambda_{5}$ is the root of (48), and

$$
\frac{\partial \lambda_{5}}{\partial t}=\frac{1}{-\frac{\lambda_{5} y}{\beta_{1}}-\frac{2 \lambda_{5} h}{\alpha_{1}}-x}
$$

$T_{P S}$ denotes the time at which the reflected shear wave induced by the incident longitudinal wave arrives. The deformed integration path for this case is similar to Fig. 2a. The intersection of the deformed path with the real axis is always located in the region between $-a_{1}$ and $a_{1}$.

Now let us consider the sixth term of (37). When we let

$$
\beta_{1}(y+2 h)-\lambda x=t
$$

(51) can be solved for $\lambda$ explicitly to yield

$$
\lambda_{\Gamma_{ \pm}}\left(r_{2}, \theta_{2}, t\right)=-\frac{t}{r_{2}} \cos \theta_{2} \pm i \sqrt{\frac{t^{2}}{r_{2}^{2}}-b_{1}^{2}} \sin \theta_{2} .
$$

When $t=b_{1} r_{2}$, it indicates the time at which the reflected shear wave front generated by the incident shear wave arrives and is denoted by $T_{S S}$. The deformed integration contour is shown in Fig. 2b. If $b_{1}\left|\cos \theta_{2}\right|>a_{1}$, an additional integration path from $t=T_{H D}$ to $t=T_{S S}$ which embraces the branch cut must be considered. In this integral interval, $\lambda$ is real and is

$$
\hat{\lambda}_{H \pm}\left(r_{2}, \theta_{2}, t\right)=-\frac{t}{r_{2}} \cos \theta_{2} \pm \sqrt{b_{1}^{2}-\frac{t^{2}}{r_{2}^{2}}} \sin \theta_{2} \text { for } T_{H D}<t<T_{S S}
$$

where

$$
T_{H D}=-\sqrt{b_{1}^{2}-a_{1}^{2}}(y-h) \pm a_{1} x .
$$

This additional integration path represents the contribution from the head wave induced by the incident shear wave, the wave front of the head wave arrives at time $t=T_{H D}$. The final form of the sixth term of (37) in time domain becomes

$$
\sigma_{y y}^{(1)}(x, y, t)=\frac{\sigma_{0}}{\pi} \operatorname{Im}\left[F_{6}\left(\lambda_{6}\right) \frac{\partial \hat{\lambda}_{6}}{\partial t}\right] H\left(t-T_{S S}\right)+\frac{\sigma_{0}}{\pi} \operatorname{Im}\left[F_{6}\left(\lambda_{7}\right) \frac{\partial \lambda_{7}}{\partial t}\right] H\left(t-T_{H D}\right),
$$

where $\lambda_{6}=\lambda_{\Gamma_{+}}\left(r_{2}, \theta_{2}, t\right)$ in (52), and $\lambda_{7}=\lambda_{H_{+}}\left(r_{2}, \theta_{2}, t\right)$ in (53). 
The inverse Laplace transform of the refracted part in the lower medium (medium 2) can be evaluated in the same manner as we have discussed for medium 1 previously. The equations of wave fronts for the first four refracted waves, i.e., the refracted longitudinal wave induced by the incident longitudinal wave $(P / P$ wave $)$, the refracted longitudinal wave induced by the incident shear wave $(S / P$ wave), the refracted shear wave induced by the incident longitudinal wave ( $P / S$ wave), and the refracted shear wave induced by the incident shear wave $(S / S$ wave), will be discussed in detail. We introduce Cagniard contours by setting

$$
\begin{aligned}
& P / P \text { wave: } \quad-\alpha_{2} y+2 \alpha_{1} h-\lambda x=t, \\
& \text { S/P wave: }-\alpha_{2} y+2 \beta_{1} h-\lambda x=t, \\
& P / S \text { wave: }-\beta_{2} y+2 \alpha_{1} h-\lambda x=t, \\
& S / S \text { wave: }-\beta_{2} y+2 \beta_{1} h-\lambda x=t .
\end{aligned}
$$

When the imaginary part of the roots in (56)-(59) vanishes, the corresponding time is denoted by $T_{P / P}, T_{S / P}, T_{P / S}$, and $T_{S / S}$, respectively. The refracted wave fronts for $P / P, S / P$, $P / S$, and $S / S$ wave will arrive at the time $T_{P / P}, T_{S / P}, T_{P / S}$, and $T_{S / S}$, respectively. The wave fronts for refracted waves are not cylindrical waves. For the case (i.e., $a_{1}<a_{2}<b_{1}<b_{2}$ ) we consider, the vertexes of curves given by (56) and (58) are always located on the real axis between the branch points at $-a_{1}$ and $a_{1}$. However, the vertexes of curves given by (57) and (59) may intersect the branch cut, which will induce head waves. The transient solutions for refracted waves in time domain are

$$
\begin{aligned}
& P / P_{\text {wave }}: \sigma_{y y}^{(2)}(x, y, t)={ }_{0}^{\sigma_{0}} \operatorname{Im}\left[F_{1}^{*}\left(\lambda_{1}^{*}\right) \frac{\partial \lambda_{1}^{*}}{\partial t}\right] H\left(t-T_{P / P}\right), \\
& S / P \text { wave }: \quad \sigma_{y y}^{(2)}(x, y, t)=\frac{\sigma_{0}}{\pi} \operatorname{Im}\left[F_{2}^{*}\left(\lambda_{2}^{*}\right) \frac{\partial \lambda_{2}^{*}}{\partial t}\right] H\left(t-T_{S / P}\right), \\
& P / S \text { wave: } \quad \sigma_{y y}^{(2)}(x, y, t)=\frac{\sigma_{0}}{\pi} \operatorname{Im}\left[F_{3}^{*}\left(\lambda_{3}^{*}\right) \frac{\partial \lambda_{3}^{*}}{\partial t}\right] H\left(t-T_{P / S}\right), \\
& S / S \text { wave: } \quad \sigma_{y y}^{(2)}(x, y, t)=\frac{\sigma_{0}}{\pi} \operatorname{Im}\left[F_{4}^{*}\left(\lambda_{4}^{*}\right) \frac{\partial \lambda_{4}^{*}}{\partial t}\right] H\left(t-T_{S / S}\right) .
\end{aligned}
$$

The values of $\lambda_{1}^{*}, \lambda_{2}^{*}, \lambda_{3}^{*}$, and $\lambda_{4}^{*}$ are the roots of (56) (59), respectively. The transient stress solutions due to the contributions from other waves can also be constructed by the procedure described above for the first few waves propagating in the two layered medium. The exact transient solutions for stress $\sigma_{y y}$ in solid 1 and solid 2 are

$$
\begin{gathered}
\sigma_{y y}^{(1)}(x, y, t)=\frac{\sigma_{0}}{\pi} \sum_{i=1}^{\infty} \operatorname{Im}\left[F_{i}\left(\lambda_{i}\right) \frac{\partial \lambda_{i}}{\partial t}\right] H\left(t-T_{i}\right)+\text { Head waves, } \\
\sigma_{y y}^{(2)}(x, y, t)=\frac{\sigma_{0}}{\pi} \sum_{i=1}^{\infty} \operatorname{Im}\left[F_{i}^{*}\left(\lambda_{i}^{*}\right) \frac{\partial \lambda_{i}^{*}}{\partial t}\right] H\left(t-T_{i}^{*}\right)+\text { Head waves. }
\end{gathered}
$$

The first few terms for the functions $F_{i}$ and $F_{i}^{*}$ were expressed previously. The number of solutions for head waves in (64)-(65) depends on the relative magnitudes of the slownesses for the first and second layers. 
For the numerical examples, a two layered composite medium is considered. The ratio of the thickness for the two layered medium is chosen to be $h_{2} / h_{1}=0.67$. At time $t=0$, an inplane dynamic concentrated loading with unit magnitude is applied at the top surface of the first layer. The time dependence of the loading is given by the Heaviside step function $H(t)$. The wave fronts in the two layered medium at the observation time $t / a_{1} h=1.4$ are shown in Fig. 3. In this diagram, the ratio of the slownesses is set to be $a_{1}: b_{1}: a_{2}: b_{2}=2: 3: 4: 5$. Only the reflected waves (i.e. $P$ wave, $S$ wave, $P P$ wave, $S S$ wave, $P P P$ wave, and $S S S$ wave) in the first layer are represented by cylindrical curves, the head wave is represented by an inclined straight line, and remaining wave fronts are represented by smooth curves which are evaluated numerically. The transient normal stress $\sigma_{y y}$ at different locations in the two layered medium is presented in Figs 4 and 5. Figure 4 shows the transient stress history at two receivers, one is at the interface $(y / h=-0.6)$ and the other $(y / h=-0.42)$ is located inside the first layer. The time has been normalized by dividing by $a_{1} h\left(h=h_{1}+h_{2}\right)$ and the arrival time for each wave front is indicated in the figure. In Fig. 5, the transient responses at the receiver located in the second layer $(y / h=-0.75)$ and at the receiver located at the interface $(y / h=0.6)$ are presented. Note

$\begin{array}{llrll}\text { 1. } & P & 8 . & P / P & 15 . P S S \\ 2 . & S & 9 . & P / S & 16 . S P P \\ 3 . & H & 10 . & S / P & 17 . S P S \\ 4 . & P P & 11 . & S / S & 18 . P / P P \\ 5 . & P S & 12 . & P P P & 19 . P / P S \\ 6 . & S P & 13 . & P P S & 20 . H H 182 \\ 7 . & S S & 14 . & P S P & 21 . H H 3 \& 4\end{array}$

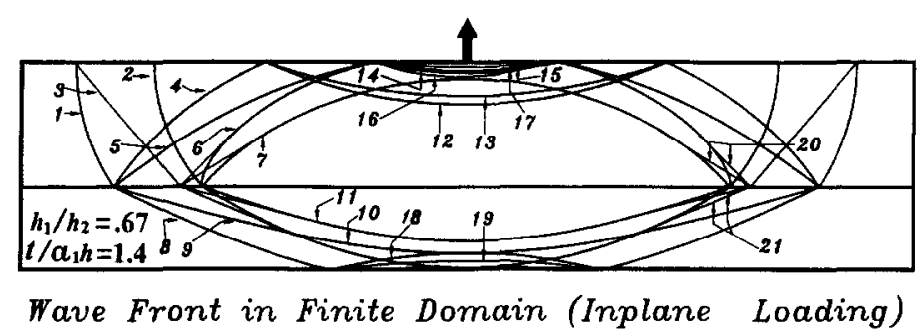

Fig. 3. Wave fronts of the incident, reflected, and refracted waves in a two layered medium for $a_{1}: b_{1}: a_{2}: b_{2}=2: 3: 4: 5$.

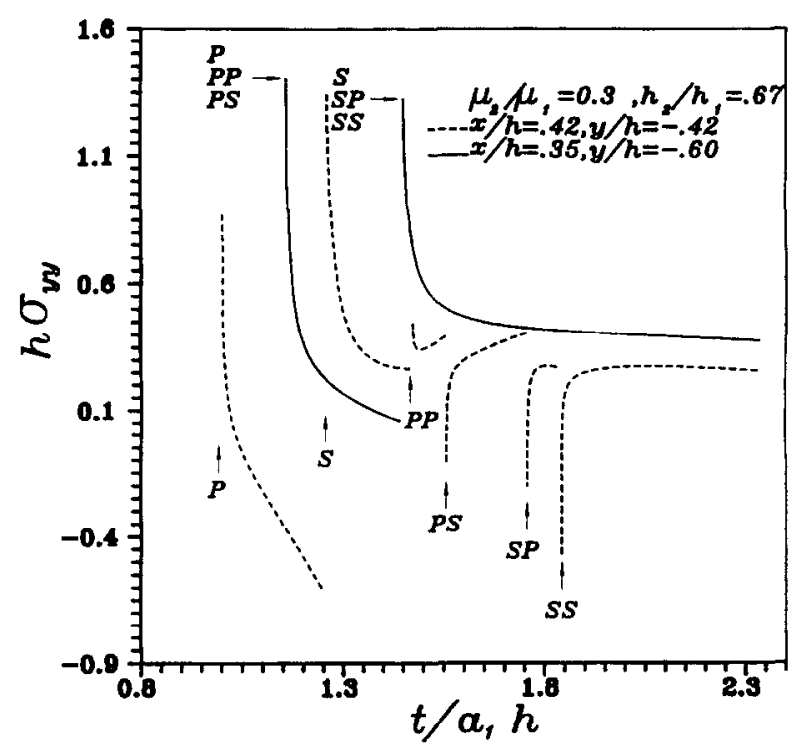

Fig. 4. Transient stress history at the receiver located in layer 1 and at the interface. 


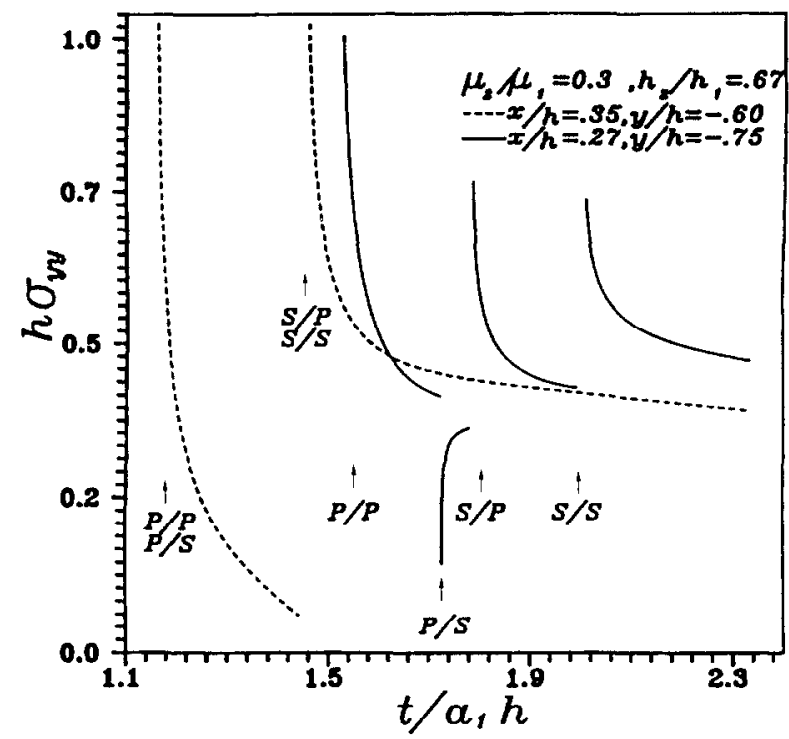

Fig. 5. Transient stress history at the receiver located in layer 2 and at the interface.

that, for the point force of Heaviside function dependence, the stress fields behave as the square root singularity for all the reflected and refracted wave fronts.

\section{CONCLUSION}

Most of the studies for wave propagation in a layered medium focus on the determination of the dispersion relation or the frequency equation due to a harmonic oscillation (Sun et al. (1968), Stern et al. (1971), Drumheller and Bedford, (1973)). Because of the difficulty in analyzing the transient response in a finite layered medium, only a few solutions based on the method of generalized ray (Pao and Gajewski (1977), Borejko and Ziegler $(1991,1993)$ ) were obtained for the transient waves in a multilayered solid. In this study, a general methodology is proposed to construct the transient solutions of a layered composite medium subjected to dynamic impact loadings generating infinite number of nonplanar reflected and refracted waves in each layer. The Cagniard-de Hoop method is used to investigate the structure of the wave pattern and transient solutions in time domain. The analytical results obtained in this study are exact and are expressed by series, each term representing a transient wave. Note that the dynamic stress response in the early time of the transient period is much larger than the static value. In this time period, the stress will change radically when the reflected or the refracted wave arrives.

Acknowledgements--The financial support for the authors from the National Science Council, Republic of China, through Grant NSC 81-0422-E-002-557 to National Taiwan University is gratefully acknowledged. The authors want to express their great appreciation to the reviewer for his valuable suggestions.

\section{REFERENCES}

Achenbach, J. D. (1975). Wave Propagation in Elastic Solids, North-Holland, Amsterdam.

Aki, K. and Richards, P. G. (1980). Quantitative Seismology, 1, W. H. Freeman \& Co, San Francisco.

Borejko, P. (1987). Application of the generalized rays to transient waves in an elastic half-space due to a buried line source. Acta Mechanica 67, 79-90.

Borejko, P. and Ziegler, F. (1991). Seismic waves in layered soil: the generalized ray theory. In Structural Dynamic: Recent Advances (ed. Schueller, G. I.), pp. 52-101, Springer-Verlag, Berlin.

Borejko, P. and Ziegler, F. (1993). Seismic pulses in layered elastic media: three dimensional generalized ray theory and its numcrical implementation. In Mathematical and Numerical Aspects of Wave Propagation (eds Kleinman, R., Angell, T., Colton, D., Santosa, F. and Stakgold, I.), pp. 92-102, SIAM, Philadelphia.

Chadwick, P. and Borejko, P. (1994). Existence and uniqueness of Stoneley waves. Geophys. J. 118, 279-284.

Cagniard, L. (1939). Reflexion et Refraction des Ondes Seismiques Progressives, Cauthiers Villars, Paris; translated into English and revised by Flinn, E. A. and Dix, C. H., 1962, Reflection and Refraction of Progressive Seismic Waves, McGraw-Hill. New York. 
Chao, C. C. (1960). Dynamic response of an elastic half-space to tangential surface loadings. J. Appl. Mech. 27, $559-567$.

de Hoop, A. T. (1960). A modification of Cagniard's method for solving seismic pulse problems. Appl. Scientific Res. 8, 349-356.

Drumheller, D. S. and Bedford, A. (1973). On a continuum theory for a laminated medium. J. Appl. Mech. 40, 528-541.

Ewing, W. M., Jardetzky, W. and Press, F. (1957). Elastic Waves in Layered Media, McGraw-Hill, New York.

Garvin, W. W. (1956). Exact transient solution of the buried line source problem. Proc. Royal Society (London) A234, 528-541.

Hegemier, G. A. and Nayfeh, A. H. (1973). A continuum theory for wave propagation in laminated composites, case 1 : propagation normal to the laminates. J. Appl. Mech. 40, 503-510.

Hegemier, G. A. and Bache, T. C. (1973). A continuum theory for wave propagation in laminated composites, case 2 : propagation parallel to laminates. $J$. Elasticity $3,125-140$.

Lamb, H. (1904). On the propagation of tremors over the surface of an elastic solid. Philosophical Trans. Royal Society (London) A203, $1-42$

Ma, C. C. and Huang, K. C. (1996). Exact transient solutions of buried dynamic point forces for elastic bimaterials Int. J. Solids Structures (in press).

Miklowitz, J. (1978). The Theory of Elastic Waves and Waveguides, North-Holland, Amsterdam.

Mukunoki, I. and Ting, T. C. T. (1980). Transient wave propagation normal to the layering of a finite layered Inedium. Int. J. Solids Structures 16, 239-251.

Nemat-Nasser, S. and Yamada, M. (1981). Harmonic waves in layered transversely isotropic composites. J. Sound and Vibration 79, 161-170.

Pckeris, C. L. (1940). A pathological case in the numerical solution of integral equations. Proc. Nat. Academy of Sciences of United States 26, 433-437.

Pekeris, C. L. (1955a). The seismic surface pulse. Proc. Nat. Academy of Sciences of United States 41, 469-480.

Pekeris, C. L. (1955b). The seismic buried pulse. Proc. Nat. Academy of Sciences of United States 41, 629639.

Pekeris, C. L. and Lifson, H. (1957). Motion of the surface of a uniform elastic half-space produced by a buried pulse. J. Acoust. Soc. Am. 29, 1233-1238.

Pekeris, C. L. and Longman, I. M. (1958). The motion of the surface of a uniform elastic half-space produced by a buried torque-pulse. Geophys. J. 1, 146-153.

Pao, Y. H. and Gajewski, R. R. (1977). The generalized ray theory and transient responses of layered elastic solids. In Physical Acoustirs 13 (eds Mason, W. P. and Thurston, R. N.), pp. 183-265, Academic Press, New York.

Stern, M., Bedford, A. and Yew, C. H. (1971). Wave propagation in viscoelastic laminates. J. Appl. Mech. 38, 448-454.

Sun, C. T., Achenbach, J. D. and Herrmann, G. (1968). Time-harmonic waves in a stratified medium propagating in the direction of the layering. J. Appl. Mech. 35, 408-411.

Tang, Z. and Ting, T. C. T. (1985). Transient waves in a layered anisotropic elastic medium. Proc. R. Soc. Lond. A397, 67-85.

Ting, T. C. T. and Mukunoki, I. (1979). A theory of viscoelastic analogy for wave propagation normal to the layering of a layered medium. J. Appl. Mech. 46, 329-336.

Tsai, C. H. and Ma, C. C. (1991). Exact transient solutions of buried dynamic point forces and displacement jumps for an elastic half space. Int. J. Solids Structures 28, 955-974. 